\title{
Luminosity studies in a traveling waist regime in the Compact Linear Collider
}

\author{
Javier Barranco García, * Eduardo Marín Lacoma, and Rogelio Tomás García \\ CERN, Geneva 23, CH-1211, Switzerland \\ (Received 27 August 2012; published 4 April 2013)
}

\begin{abstract}
The final focus system of the Compact Linear Collider (CLIC) features a crab cavity just before the final quadrupole doublet to ensure head-on collisions at the interaction point. A decrease in the expected luminosity with respect to the case without crossing angle has been recently observed in simulations for the CLIC baseline configuration. This effect is explained from the aberrations induced due to a $z$-dependent off-center horizontal orbit in the final focus sextupoles produced by the crab cavity. Three options to recover from this loss are explored. The effect of the longitudinal $E-z$ correlation of the incoming beam from the linac is taken into account and its effect on the expected luminosity is evaluated.
\end{abstract}

DOI: 10.1103/PhysRevSTAB.16.041001

PACS numbers: 29.20.Ej, 41.75.Ht

\section{INTRODUCTION}

A horizontal crossing angle $\left(\theta_{c}\right)$ between the beams at the interaction point (IP) is introduced in the Compact Linear Collider (CLIC) beam delivery system (BDS) [1] to minimize effects of parasitic crossing of bunches and to extract the spent beam cleanly. This crossing scheme produces a loss of geometric luminosity with respect to the zero crossing angle collision according to

$$
\mathcal{L} \approx \mathcal{L}_{\theta_{\mathrm{c}}=0} \frac{1}{\sqrt{1+\Theta^{2}}}
$$

where $\Theta$ is the Piwinski angle,

$$
\Theta \equiv \frac{\tan \left(\theta_{c} / 2\right) \sigma_{z}}{\sigma_{x}}
$$

where $\theta_{c}$ is the full crossing angle, $\sigma_{z}$ the bunch length, and $\sigma_{x}$ the horizontal beam size. Considering the CLIC parameters (Table I), $\Theta \approx 10$ and thus $\mathcal{L} / \mathcal{L}_{\theta_{\mathrm{c}}=0} \sim 10 \%$. In addition, the enhancement factor that takes into account the effect of the disruptive fields, i.e., Pinch effect, is reduced as the focusing effect of one beam on the other is minimized in the presence of a crossing angle. This translates into an even larger loss of luminosity. To recover head-on collisions with a certain crossing angle, Palmer introduced the concept of crab cavities (CC) [3]. The CLIC final focus baseline design follows the novel final focus concept proposed in [4], including a single crab cavity just before the final doublet (FD) as shown in Fig. 1.

A crab cavity is a deflection cavity operated with a $90^{\circ}$ phase shift (crabbing), so the center of each bunch remains undeflected, whereas the head is kicked to one side and the tail to the other. The total effect is a tilt of $\theta_{c} / 2$ with respect

\footnotetext{
*javier.barranco@cern.ch
}

Published by the American Physical Society under the terms of the Creative Commons Attribution 3.0 License. Further distribution of this work must maintain attribution to the author(s) and the published article's title, journal citation, and DOI. to the uncrabbed motion. In addition to preserve symplecticity a kick in energy is applied dependent on the transverse position. If the bunch length is much shorter than the wavelength of the $\operatorname{rf}\left(\lambda \gg \sigma_{z}\right)$, the kick will lie in the linear part. Therefore, it can be approximated by

$$
\Delta x^{\prime}(z)=\xi_{c} z, \quad \Delta \delta(x)=\xi_{c} x,
$$

with $\xi_{c}$,

$$
\xi_{c}=\frac{\omega_{\mathrm{rf}} q V_{\mathrm{CC}}}{c \quad E_{0}}
$$

and $\omega_{\mathrm{rf}}$ the rf frequency, $c$ the speed of light, $q$ the particle charge, $V_{\mathrm{CC}}$ the crab cavity voltage, and $E_{0}$ the nominal energy. Similarly, the voltage needed in the crab cavity depends on the $R_{12}$ matrix element between the crab cavity and the IP, and the rf frequency,

$$
V_{\mathrm{CC}}=\frac{\frac{\theta_{c}}{2} E_{0} c}{R_{12}^{\mathrm{CC}-\mathrm{IP}} q \omega_{\mathrm{rf}}}
$$

The sign of the tilts is such that the two bunches are in line during collision. In their own center of mass, they interact with zero crossing angle and a priori they suffer no luminosity loss [3]. Nevertheless, some luminosity degradation has been recently first observed in simulations for

TABLE I. Nominal parameters for the CLIC BDS version 10_01_25 [2].

\begin{tabular}{lcc}
\hline \hline Parameter & Units & Value \\
\hline Crossing angle at the IP & $\mathrm{mrad}$ & 20 \\
Nominal core beam size at the IP $\left(\sigma_{x / y}^{*}\right)$ & $\mathrm{nm}$ & $45 / 1$ \\
Nominal beam divergence at IP $\left(\theta_{x / y}^{*}\right)$ & $\mu \mathrm{rad}$ & $7.7 / 10.3$ \\
Nominal beta function at IP $\left(\beta_{x / y}^{*}\right)$ & $\mathrm{mm}$ & $10 / 0.07$ \\
Nominal bunch length $\left(\sigma_{z}\right)$ & $\mu \mathrm{m}$ & 44 \\
Nominal energy spread flat beam $\left(\sigma_{\delta}\right)$ & $\%$ & 1 \\
Maximum energy/beam & $\mathrm{TeV}$ & 1.5 \\
rf frequency & $\mathrm{GHz}$ & 12 \\
Train repetition rate & $\mathrm{Hz}$ & 50 \\
\hline \hline
\end{tabular}




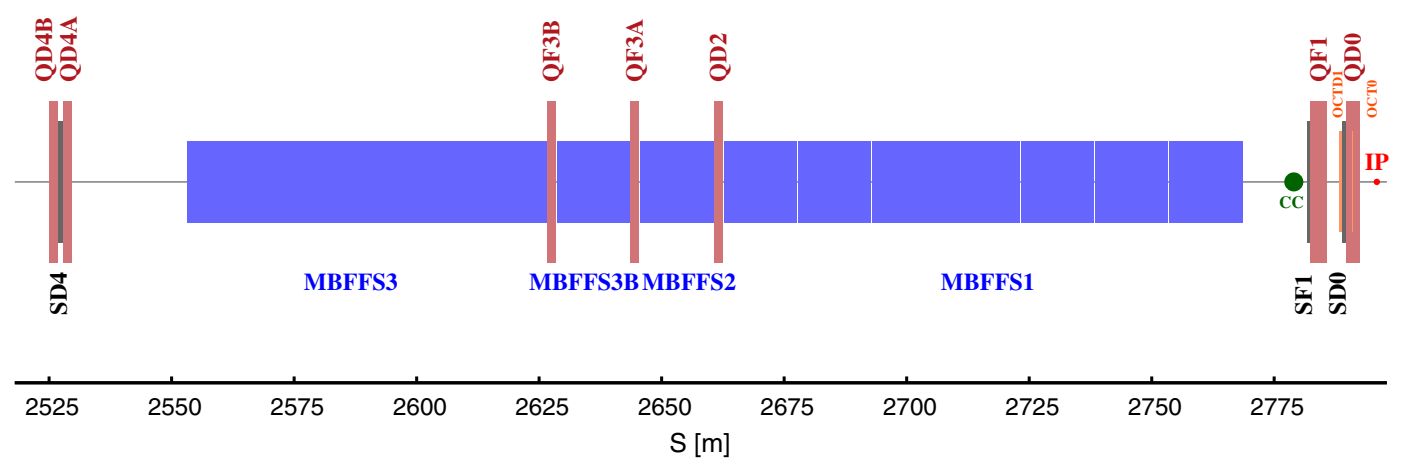

FIG. 1. Layout of the elements in the last part of the CLIC BDS. The crab cavity is located just before the final doublet. After being deflected by the crab cavity the beam will pass horizontally off center in the two last sextupoles, SF1 and SD0.

the CLIC baseline configuration [5]. The reasons for the loss of luminosity are explored and some possible cures are proposed.

\section{LUMINOSITY LOSS IN A CRAB CAVITY SCHEME}

In the nominal scenario (Fig. 1), the transfer matrix element between the crab cavity and the IP is $R_{12}=$ 23.04. This corresponds to a necessary crab cavity voltage of $V_{\mathrm{CC}}=2.6 \mathrm{MV}$ to ensure a $10 \mathrm{mrad}$ beam rotation at the IP. PLACET + GUINEA PIG (P + GP) [6,7] simulations for the 10_01_25 version of the CLIC BDS lattice showed a loss of luminosity $\Delta \mathcal{L} / \mathcal{L}_{\theta_{\mathrm{c}}=0}=9.4 \%$ with respect to the zero crossing angle case.

After the crab cavity has introduced an horizontal divergence, the beam passes off center in the horizontal plane through the two sextupoles in the FD, SF1, and SD0. This horizontal displacement is linear with $z$, as shown in Eq. (3), and implies a different quadrupolar component fed down in each sextupole according to the longitudinal position of the particle within the bunch. This focusing dependence on the longitudinal position will be translated into a waist of the bunch that moves longitudinally during the collision process, or traveling waist regime [8]. The waist displacement at the IP due to an off-center trajectory through a single sextupole is given by

$$
w_{y}(z)=-\beta_{y} \beta_{y}^{*} R_{12}^{\mathrm{CC}-\operatorname{sext}} \xi_{c} z K L,
$$

where $\beta_{\mathrm{y}}$ is the $\beta$ function at the sextupole location, $\beta_{\mathrm{y}}^{*}$ is the $\beta$ function at the IP, $R_{12}^{\mathrm{CC}-\text { sext }}$ is the transfer matrix element from the crab cavity to the sextupole, $\xi_{c} z$ is the transverse kick as in Eq. (3), $K$ the sextupole strength, and $L$ is the sextupole length. However, this expression remains an approximation as it considers the phase advance between sextupoles and the IP to be $(2 n+1) \pi / 2$ as in the lattice used in this study.

Likewise, the variation of the waist displacement with respect to the longitudinal position within the bunch is linear and the gradient or traveling waist parameter can be obtained by differentiating Eq. (6):

$$
\frac{\partial w_{y}}{\partial z}=-\beta_{y}^{*} \sum_{i}^{n_{s}} R_{12}^{\mathrm{CC}-\operatorname{sext}_{i}} \beta_{y_{i}} \xi_{c} K_{i} L_{i}
$$

where $n_{s}$ is the total number of sextupoles between the crab cavity and the IP.

The sign of Eq. (7) indicates the evolution of the waist during the collision process. In order to maximize luminosity, the beam waist should be at the slice which collides with the center of the opposite bunch at a certain longitudinal position $s$. We refer to that particular slice as colliding slice. For negative traveling waist parameters the colliding slice is defocused when it collides with the center of the other bunch, producing a loss in luminosity. In the current crossing scheme with $\theta_{c} / 2=+10 \mathrm{mrad}$ the tracking simulations reveal a $\left(y \mid y_{0}^{\prime} z_{0}\right)$ correlation at the IP in the form of traveling waist of magnitude $\frac{\partial w_{y}}{\partial z}=-1.14$. This correlation is better seen in Fig. 2 where the vertical beam size of the colliding slice is plotted at different longitudinal positions around the IP. For the case without crossing angle and no crab cavity (blue trace), the vertical beam size follows the beta function around the IP or hourglass effect

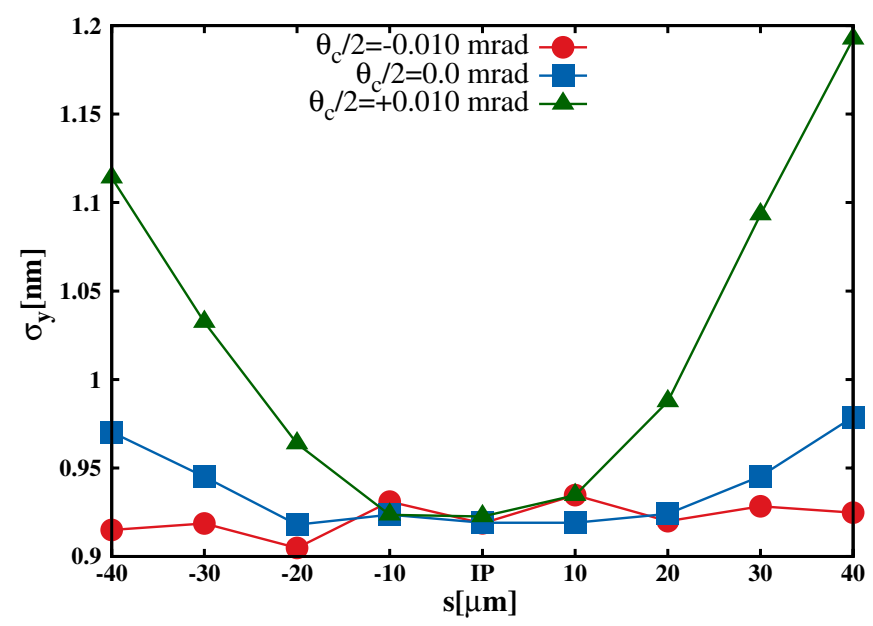

FIG. 2. Vertical beam size of the colliding slice at different longitudinal locations before and after the IP for an initial distribution with no $E-z$ correlation from the linac. 
with a minimum at the IP. In the nominal scheme with $\theta_{c} / 2=+0.010 \mathrm{mrad}$ (green trace), the beam size of the colliding slice is increased as explained before.

The presence of a traveling waist is confirmed, in addition, by the analysis of the map coefficients that contribute to the vertical beam size increase. Simulations done with PTC and MAPCLASS [9] found that the term $T_{\mathrm{yyz}}$ is the main contributor to the vertical beam size growth. As well, higher order nonlinear terms like $U_{\text {yyzz }}$ are also found to contribute to the increase [10].

Three possible options to recover from this loss are evaluated next.

\section{A. Two crab cavities scheme}

This option is analyzed for historical reasons as it was proposed for The International Linear Collider (ILC) as a possible way to correct the loss of luminosity [11]. The addition of a second crab cavity with opposite polarity is used to compensate the increase of vertical beam size. However, this solution presents the side effect of a horizontal beam size distortion. For the CLIC configuration only a second crab cavity in the same long drift $\Delta L=$ $11.3 \mathrm{~m}$ has been considered. An optimum solution was found with the second crab cavity at a distance $d=5 \mathrm{~m}$ from the first. The optimized voltages are in this case $V_{\mathrm{CC}, 1}=5 \mathrm{MV}$ and $V_{\mathrm{CC}, 2}=-2.52 \mathrm{MV}$, recovering only up to $\mathcal{L} / \mathcal{L}_{\theta_{\mathrm{c}}=0} \sim 93.4 \%$. In this case the tracked distribution reveals a traveling parameter of $\frac{\partial w_{y}}{\partial z}=-0.84$. The analytical calculation for this case is done by extending the equation to include additional crab cavities,

$$
\frac{\partial w_{y}}{\partial z}=-\beta_{y}^{*} \sum_{i}^{n_{s}} \sum_{j}^{n_{c c}} R_{12}^{\mathrm{CC}_{j}-\operatorname{sext}_{i}} \beta_{y_{i}} \xi_{c} K_{i} L_{i}
$$

Evaluating the previous expression we obtain $\frac{\partial w_{y}}{\partial z}=$ -0.86 very close to the simulated result. Nevertheless, the technical issues associated with the operation of two crab cavities make this option one of the less preferred.

\section{B. Crab cavity displacement}

As already explained, the traveling waist regime depends on the relative position between the crab cavity and the sextupoles downstream. For this reason the traveling waist parameter and the luminosity loss are evaluated for new locations for a single crab cavity scheme. The CLIC crab cavities are compact devices of $L_{\mathrm{cc}}=0.3 \mathrm{~m}$ [2] that can be easily accommodated in different locations. Four different options are considered: (1) between QF1 and SD0 $(\Delta L=3 \mathrm{~m})$ : (a) before octupole OCT0 and (b) after octupole OCT0; (2) between SF1 and QF1 ( $\Delta L=0.25 \mathrm{~m}$, if necessary the magnets could be slightly displaced to accommodate the crab cavity without major impact on the optics); (3) between QD4B-SD4 and SF5 ( $\Delta L=8 \mathrm{~m})$.

The traveling waist parameters are calculated again with two approaches, first evaluating the analytical formula in Eq. (7) and second as a fit of a tracked distribution with $\mathrm{P}+\mathrm{GP}$. The results summarized in Table II for the different cases show an extremely good agreement between both. Two locations recover almost fully the luminosity with respect to the zero crossing scheme case, before SD0 and before SD4. In the first case, the traveling waist parameter is only $\frac{\partial w_{y}}{\partial z}=-0.12$ due to the small $R_{12}$ between $\mathrm{CC}$ and SD0. In the second case, the contributions from the three sextupoles SF4, SF1, and SD0 cancel each other leading to a traveling waist parameter of $\frac{\partial w_{y}}{\partial z}=+0.25$. The positive sign indicates that in this case the waist moves from the head to the tail.

Nevertheless, there are some practical limitations when selecting the position of the crab cavity. Two things have to be considered: (i) the distance to the IP, and (ii) the voltage needed in the crab cavity. Regarding (i), both cavities, one per line, will be fed by the same klystron placed at the IP. At further distances higher power from the klystron might be needed requiring an additional klystron or pulse compression. As well, the synchronization system might be reviewed. This can be an issue for case (3). For (ii), this value is inversely proportional to $R_{12}^{\mathrm{CC}-\mathrm{IP}}$ as in Eq. (5), so in locations like case (1a) the high voltage needed can be an issue.

TABLE II. Traveling waist and luminosity variation for different locations of the crab cavity in the CLIC BDS. For each location the luminosity is optimized by scanning the voltage in the crab cavity using P + GP. The traveling waist is evaluated both with the analytical formula in Eq. (7) and an empirical fit from tracking simulations. The luminosity is calculated by P + GP simulations, applying Eq. (9) to a zero crossing angle distribution at the IP and P + GP simulations with $E-z$ correlated distribution from the linac.

\begin{tabular}{lccccccc}
\hline \hline Case & $\begin{array}{c}R_{12}^{\mathrm{CC}-\mathrm{IP}}[\mathrm{m}] \\
(\mathrm{MADX}-\mathrm{PTC})\end{array}$ & $\begin{array}{c}V_{\mathrm{CC}}[\mathrm{MV}] \\
(\mathrm{P}+\mathrm{GP})\end{array}$ & $\begin{array}{c}\partial w_{y} / \partial z[-] \\
(\text { Analytical })\end{array}$ & $\begin{array}{c}\partial w_{y} / \partial z[-] \\
(\mathrm{P}+\mathrm{GP})\end{array}$ & $\begin{array}{r}\mathcal{L} / \mathcal{L}_{\theta_{\mathrm{c}}=0}[\%] \\
(\mathrm{GP})\end{array}$ & $\begin{array}{c}\mathcal{L} / \mathcal{L}_{\theta_{\mathrm{c}}=0} \\
{[\%]}\end{array}(\mathrm{P}+\mathrm{GP})$ & $\begin{array}{c}\mathcal{L} / \mathcal{L}_{\theta_{\mathrm{c}}=0}[\%] \\
(E-z)(\mathrm{P}+\mathrm{GP})\end{array}$ \\
\hline $2 \mathrm{CC}$ & $23.06 / 22.19$ & $5.0 /-2.52$ & -0.86 & $-0.84 \pm 0.01$ & 93.9 & 93.4 \\
Nominal $\left(\theta_{c} / 2>0\right)$ & 23.06 & 2.60 & -1.15 & $-1.14 \pm 0.01$ & 91.1 & 90.6 & 93.7 \\
1a & 10.33 & 5.68 & -0.12 & $-0.12 \pm 0.01$ & 99.5 & 98.9 & 99.2 \\
1b & 17.86 & 3.31 & -0.67 & $-0.68 \pm 0.01$ & 95.6 & 94.5 & 94.4 \\
2 & 23.54 & 2.54 & -0.99 & $-1.01 \pm 0.01$ & 92.2 & 92.1 & 92.5 \\
3 & -13.05 & -4.58 & +0.29 & $+0.25 \pm 0.01$ & 99.0 & 101.0 & 100.3 \\
Nominal $\left(\theta_{c} / 2<0\right)$ & 23.06 & -2.60 & +1.15 & $+1.14 \pm 0.01$ & 100.4 & 100.3 \\
\hline \hline
\end{tabular}




\section{Different crossing scheme}

The loss of luminosity has been explained due to the opposite evolution of the traveling waist with respect to the collision process. According to Eq. (7) it is possible to revert the direction of the traveling waist by adopting a crossing scheme of $\theta_{c} / 2=-10 \mathrm{mrad}$ while keeping the current optics in the BDS. If the polarity of the sextupoles SF1 and SD0 remains unchanged and $V_{\mathrm{cc}}$ changes due to a change of crossing scheme then $\frac{\partial w_{y}}{\partial z}$ becomes positive. The colliding slice will be then focused when colliding with the center of the opposite bunch (Fig. 2, red trace). The proposed crossing scheme with $\theta_{c} / 2=-10 \mathrm{mrad}$ is symmetric $\left(\frac{\partial w_{y}}{\partial z}=+1.14\right)$ with respect to the nominal baseline $\left(\frac{\partial w_{y}}{\partial z}=-1.14\right)$.

The luminosity dependence on the traveling waist and waist shift is shown in Fig. 3. The luminosities are calculated with GUINEA PIG, starting from a head-on distribution at the IP transformed using the following relation:

$$
y=y_{0}+\frac{\partial w_{y}}{\partial z} z_{0} y_{0}^{\prime}+w_{y} y_{0}^{\prime},
$$

where $\frac{\partial w_{y}}{\partial z}$ is the traveling waist and $w_{y}$ is the waist shift. Figure 3 shows how by changing the crossing scheme the working point moves towards a more stable region where the luminosity loss is avoided. The luminosity is recovered up to $100.3 \%$ of $\mathcal{L}_{\theta_{\mathrm{c}}=0}$. The results in Fig. 3 show that for the case of a traveling waist regime from tail to head, i.e. $\frac{\partial w_{y}}{\partial z}<0$, the luminosity degrades rapidly for decreasing values of $\frac{\partial w_{y}}{\partial z}$. On the other hand, for $\frac{\partial w_{y}}{\partial z}>0$ the variation is much less. This is explained from the fact that the maximum luminosity region is centered around $\frac{\partial w_{y}}{\partial z}=$
+0.5 . In addition, this result suggests that the lattice should be tuned with a waist shift $w_{y} \sim 25 \mu \mathrm{m}\left(\sim 0.56 \sigma_{z}\right)$ to lie in the center of the $100 \% \mathcal{L}_{\theta_{\mathrm{c}}=0}$ isoline. This is consistent with results obtained for the ILC case in [12], where the waist was proposed to be moved up to $1 \sigma_{z}$ after the IP to maximize the luminosity. As well, future studies should tackle the BDS tuning [13] in a traveling waist regime. A difference in the computed luminosity is found when comparing the values obtained after tracking in the different cases (Table II, column 7) and the ones obtained by transforming the head-on distribution at the IP according to Eq. (9) (Table II, column 6). The values in the first case are lower. The reason for this is the fact that in the second case the higher order aberrations coming from sextupoles and octupoles in the FD are not considered so resulting in smaller beam sizes. This result is consistent with the contributions to the beam size calculated by MAPCLASS [10].

In the initial crossing scheme considered as a baseline for the CLIC conceptual design report, the crossing scheme was a free parameter only constrained by the civil engineering. To revert the sign of the crossing angle, both the main linac and the BDS have to be moved rigidly together from the turn around (TA). One possibility is to redesign the basic cell of the TA so the total bending angle is decreased as shown in Fig. 4, where the present configuration (red trace) and the proposed one (blue trace) are shown. In this case the optics in the main linac and the BDS remain unchanged, so no impact in beam dump lines, machine detector interface, etc. is expected either. Future revisions of the CLIC civil engineering should take this requirement into account.

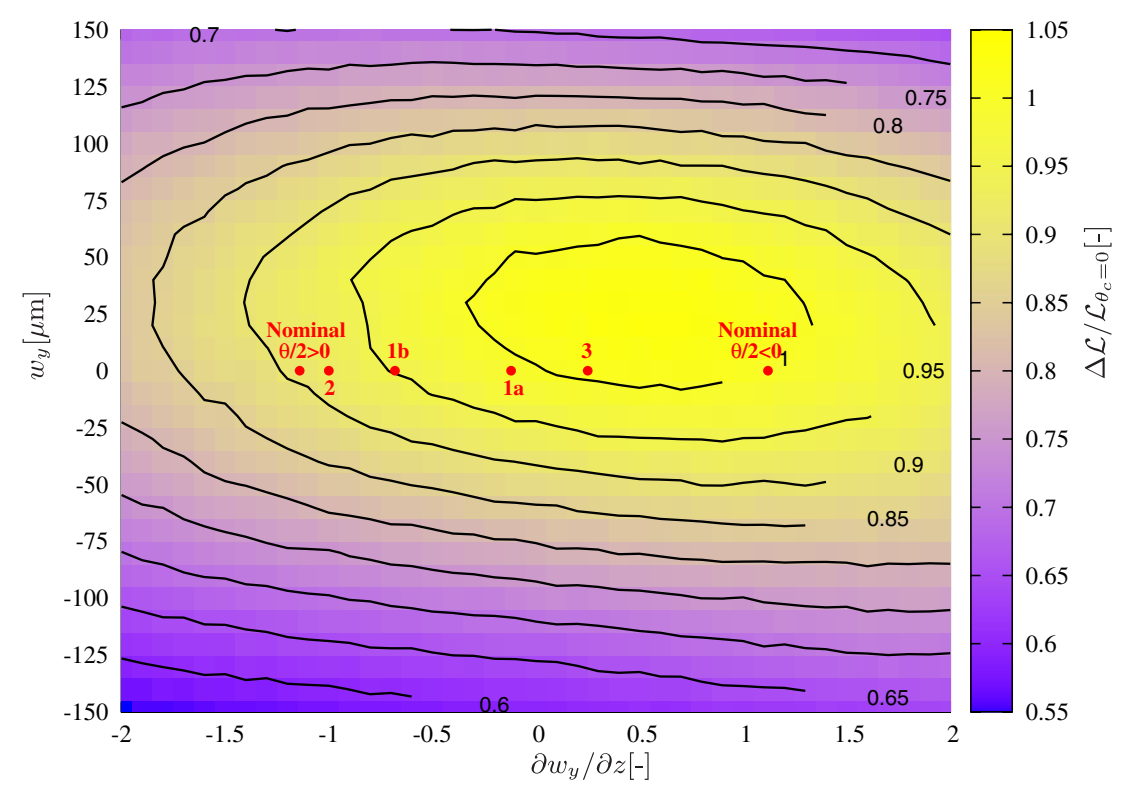

FIG. 3. Luminosity scan for different values of traveling waist $\left(\frac{\partial w_{y}}{\partial z}\right)$ and waist shift $\left(w_{y}\right)$. The luminosity is normalized with respect to the case without crossing angle and no $E-z$ correlation in the incoming beam from the linac. The different options studied are depicted with the same notation as in Table II. The maximum luminosity region corresponds to positive traveling waist parameters and is centered around a waist shift of $\sim 25 \mu \mathrm{m}$ which is consistent with results obtained for ILC [12]. 


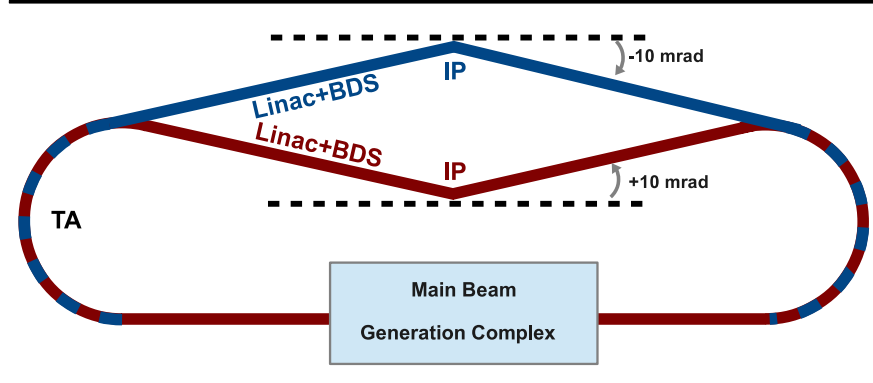

FIG. 4. Sketch (not to scale) of turn around (TA), main linac, and beam delivery system (BDS) for present (red) and proposed (blue) crossing schemes. In order to revert the sign of the crossing angle the main linac and BDS have to be rigidly moved from the TA. This can be achieved, for example, by redesigning the basic cell of the TA to decrease the total bending angle.

\section{III. $E-z$ CORRELATION}

Up to this point the incoming beam from the linac has been considered to have a flat uncorrelated energy distribution. However, due to wakefields and off-crest acceleration, the expected energy profile presents a sinusoidal-like shape where the head has a positive energy shift and the tail negative. Assuming this more realistic initial distribution, the traveling waist regime is not altered although an increase of the vertical beam size is found for slices colliding after the IP $(s>0)$ which corresponds to $\delta p / p>0$ (Fig. 5). On the other hand, this effect does not produce a loss in the relative luminosity recovery with respect to the flat energy profile but actually a slight increase. The relative luminosity change with respect to the zero crossing angle case for a $\theta_{c} / 2=-10 \mathrm{mrad}$ is $\mathcal{L} / \mathcal{L}_{\theta_{\mathrm{c}}=0, E-z}=103.3 \%$. The results presented in Table II,

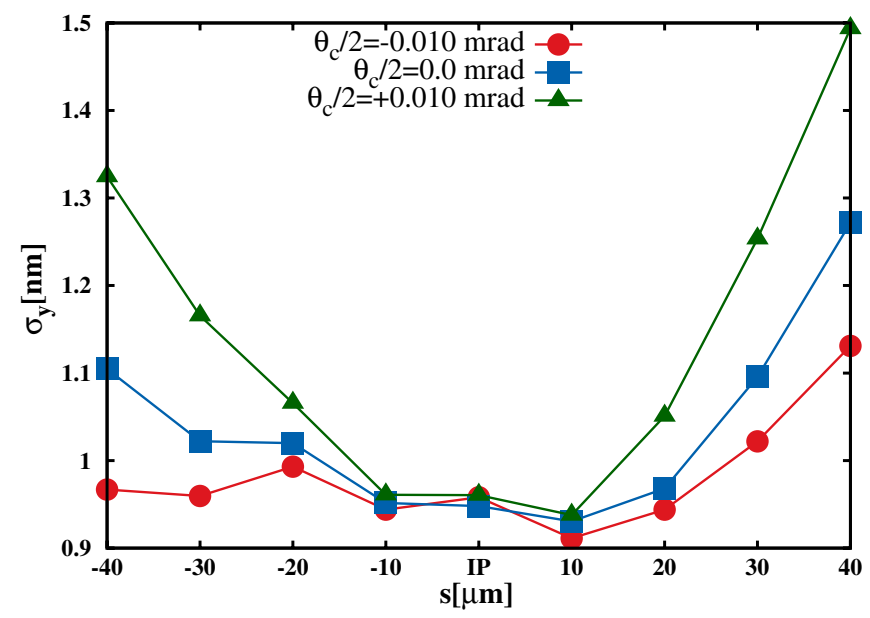

FIG. 5. Vertical beam size of the colliding slice at different longitudinal locations before and after the IP for an initial distribution with real sinusoidal-like $E-z$ correlation from the linac. last column, show comparable luminosities to the no $E-z$ correlation case.

\section{CONCLUSIONS}

The loss of luminosity in the presence of a crab cavity in the CLIC BDS has been explained with the evolution of the traveling waist. Several possibilities to mitigate or even fully compensate for this loss have been presented, with the $\theta_{c} / 2=-10 \mathrm{mrad}$ crossing scheme as the preferred option. The CLIC site civil engineering should be reviewed taking this new constraint into account. In addition a waist shift of about $25 \mu \mathrm{m}$ is proposed to maximize the luminosity. The $E-z$ correlation from the linac shows no relevant effect in the traveling waist regime or the luminosity although a distortion in the vertical beam size is found.

\section{ACKNOWLEDGMENTS}

The authors would like to acknowledge Daniel Schulte for the useful discussions, Barbara Dalena and Jochem Snuverink for their assistance with the PLACET code, and finally John Osborne for providing and helping us with the civil engineering designs.

[1] R. Tomás, Phys. Rev. ST Accel. Beams 13, 014801 (2010).

[2] M. Aicheler, P. Burrows, M. Draper, T. Garvey, P. Lebrun, K. Peach, N. Phinney, H. Schmickler, D. Schulte, and N. Toge, Technical Reports No. CERN-2012-007, No. SLAC-R-985, No. KEK-Report-2012-1, and No. PSI-12-01. JAI-2012-001, 2012.

[3] R. B. Palmer, Annu. Rev. Nucl. Part. Sci. 40, 529 (1990).

[4] Pantaleo Raimondi and Andrei Seryi, Phys. Rev. Lett. 86, 3779 (2001).

[5] I. R. R. Shinton, G. Burt, C. J. Glasman, R. M. Jones, and A. Wolski, Nucl. Instrum. Methods Phys. Res., Sect. A 657, 126 (2011).

[6] D. Schulte et al., "The tracking code placet" [https:// savannah.cern.ch/projects/placet].

[7] D. Schulte, 5th International Computational Accelerator Physics Conference, Monterey, CA, USA, 1999.

[8] V.E. Balakin, 3rd International Workshop on Linear Colliders (LC91), Protvino, USSR, 1991.

[9] R. Tomás, Phys. Rev. ST Accel. Beams 9, 081001 (2006).

[10] E. Marín Lacoma, Ph.D. thesis, Universitat Politécnica Catalunya, 2012.

[11] A. Seryi, "Crab cavity effects on y-beam size for ILC" [http://www.project.slac.stanford.edu/lc/bdir/ meetings/beamdelivery/2005-01-25/crab_25jan05.pdf].

[12] D. Schulte and R. Tomás, ICFA Beam Dyn. Newslett. 52, 149 (2010) [http://icfa-usa.jlab.org/archive/newsletter/ icfa_bd_nl_52.pdf].

[13] B. Dalena, J. Barranco, A. Latina, E. Marin, J. Pfingstner, D. Schulte, J. Snuverink, R. Tomás, and G. Zamudio, Phys. Rev. ST Accel. Beams 15, 051006 (2012). 